\title{
Quantitative myocardial perfusion in mice based on the signal intensity of flow sensitized CMR
}

\author{
Sumeda Abeykoon ${ }^{1,2}$, Michelle Sargent ${ }^{3}$ and Janaka P Wansapura ${ }^{2^{*}}$
}

\begin{abstract}
Background: In the conventional approach to arterial spin labeling in the rodent heart, the relative difference in the apparent $T_{1}$ relaxation times corresponding to selective and non-selective inversion is related to perfusion via a two compartment model of tissue. But accurate determination of $T_{1}$ in small animal hearts is difficult and prone to errors due to long scan times and high heart rates. In this study we introduce the theoretical frame work for an alternative method (SI-method) based purely on the signal intensity of slice-select and non-select inversion recovery images at a single inversion time at short repetition time.

Methods: A modified Bloch equation was solved to derive perfusion as a function of signal intensity of flow sensitized segmented gradient echo acquisitions. A two compartment fast exchanging model of tissue was assumed. To test the new technique first it was implemented on a flow phantom and then it was compared with the conventional $T_{1}$ method in an in vivo study of healthy C57BL/6 mice $(n=12)$. Finally the SI-method was used in comparison to a Late Gadolinium Enhanced (LGE) method to qualitatively and quantitatively assess perfusion deficits in an ischemia-reperfusion mouse model $(n=4)$.

Results: The myocardial perfusion of healthy mice obtained by the $S /$-method, $5.6 \pm 0.5 \mathrm{ml} / \mathrm{g} / \mathrm{min}$, (mean \pm standard deviation) was similar ( $p=0.38$ ) to that obtained by the conventional method, $5.6 \pm 0.3 \mathrm{ml} / \mathrm{g} / \mathrm{min}$. The variance in perfusion within the left ventricle was less for the SI-method than that for the conventional method ( $p<0.0001$ ). The mean percentage standard deviation among repeated measures was $3.6 \%$. The LGE regions of the ischemia reperfusion model were matched with regions of hypo-perfusion in the perfusion map. The average perfusion in the hypo perfused region among all four IR mice was $1.2 \pm 0.9 \mathrm{ml} / \mathrm{g} / \mathrm{min}$ and that of the remote region was $4.4 \pm 1.2 \mathrm{ml} / \mathrm{g} / \mathrm{min}$.
\end{abstract}

Conclusions: The proposed signal intensity based ASL method with a segmented acquisition scheme allows accurate high resolution perfusion mapping in small animals. It's short scan time, high reproducibility and ease of post process makes it a robust alternative to the conventional ASL technique that relies on $T_{1}$ measurements.

Keywords: Myocardial perfusion, T1, Mice, Arterial spin labeling

\section{Background}

To quantify perfusion, the Arterial Spin Labeling (ASL) technique uses water protons as a freely diffusible contrast agent. The contrast due to perfusion is obtained by preparation of magnetization by selective and nonselective inversion. Slice-selective inversion causes signal enhancement due to in-flow of thermally stabilized spins whereas global inversion is insensitive to flow. In the

\footnotetext{
* Correspondence: janaka.wansapura@cchmc.org

${ }^{2}$ Imaging Research Center, Department of Radiology, Cincinnati Children's

Hospital, 3333 Burnet Ave, MLC 5033, Cincinnati, OH, 45229, USA

Full list of author information is available at the end of the article
}

cardiac implementation of ASL, spin labeling is generally done within the detection slice. One approach of ASL is to mathematically relate the relative difference in the apparent relaxation times corresponding to selective and non-selective inversion, to perfusion via a two compartment model of tissue [1]. In this case perfusion, $P$, is given by:

$$
P=\frac{\lambda}{T_{1 c}}\left(\frac{T_{1 g}}{T_{1 s}}-1\right)
$$

\section{Biomed Central}

(c) 2012 Abeykoon et al.; licensee BioMed Central Ltd. This is an Open Access article distributed under the terms of the Creative Commons Attribution License (http://creativecommons.org/licenses/by/2.0), which permits unrestricted use, distribution, and reproduction in any medium, provided the original work is properly cited. 
Where, $\mathrm{T}_{\mathrm{ls}}$ and $\mathrm{T}_{\mathrm{lg}}$ are the apparent longitudinal relaxation times for slice select and non-select inversion respectively. $\mathrm{T}_{\mathrm{lc}}$ is the longitudinal relaxation time of blood. This ASL method, hereafter referred to as the T1method, has been used to quantify myocardial perfusion in humans [2,3] as well as in small animal models [4-7]. Accurate determination of $T_{1}$ relaxation times require long scan time to allow for full relaxation of the longitudinal magnetization. Long scan times in turn leads to varied inversion times for different read outs of the kspace in segmented acquisitions in the heart, causing errors in $\mathrm{T}_{1}$ maps and therefore in perfusion maps.

In this study we propose an alternative ASL method, which we will call the SI-method, based purely on the signal intensity of slice-select and nonselect inversion recovery images at a single inversion time at short repetition time. A steady state image is also acquired to normalize the receiver characteristics. A new expression for perfusion is derived for this acquisition scheme assuming a two compartment model of tissue [1]. Similar signal intensity based methods with single inversion times have been used in the past in brain and cardiac perfusion imaging [8-11]. A theoretical frame work for ASL with short repetition time has also been introduced by Pell et al. [11]. What is different in the current study is the use of short repetition time approach combined with a dedicated two-compartment theory.

We adapted the two compartment model proposed by Bauer et al. [1] and derived perfusion as a function of signal intensity of slice select and non-select inversion acquisitions by solving a modified Bloch equation. To test the new technique we first implemented it on a flow phantom with two compartments with no exchange between them. Since it is difficult to accurately quantify spin exchange rates in materials, this phantom experiment allowed us to know perfusion exactly given a specific rate of flow. Second, we compared the proposed SI-method with the conventional T1-method by carrying out in vivo myocardial perfusion measurements in healthy mice. Finally the SImethod was used in comparison to a LGE method to qualitatively and quantitatively assess perfusion deficits in an ischemia-reperfusion mouse model.

\section{Methods}

SI-method for a fast exchanging two compartment model of tissue

The data acquisition scheme for this method consists of three scans: (i) Slice selective inversion-TI-acquisition, (ii) Non-select inversion-TI-acquisition, (iii) steady state

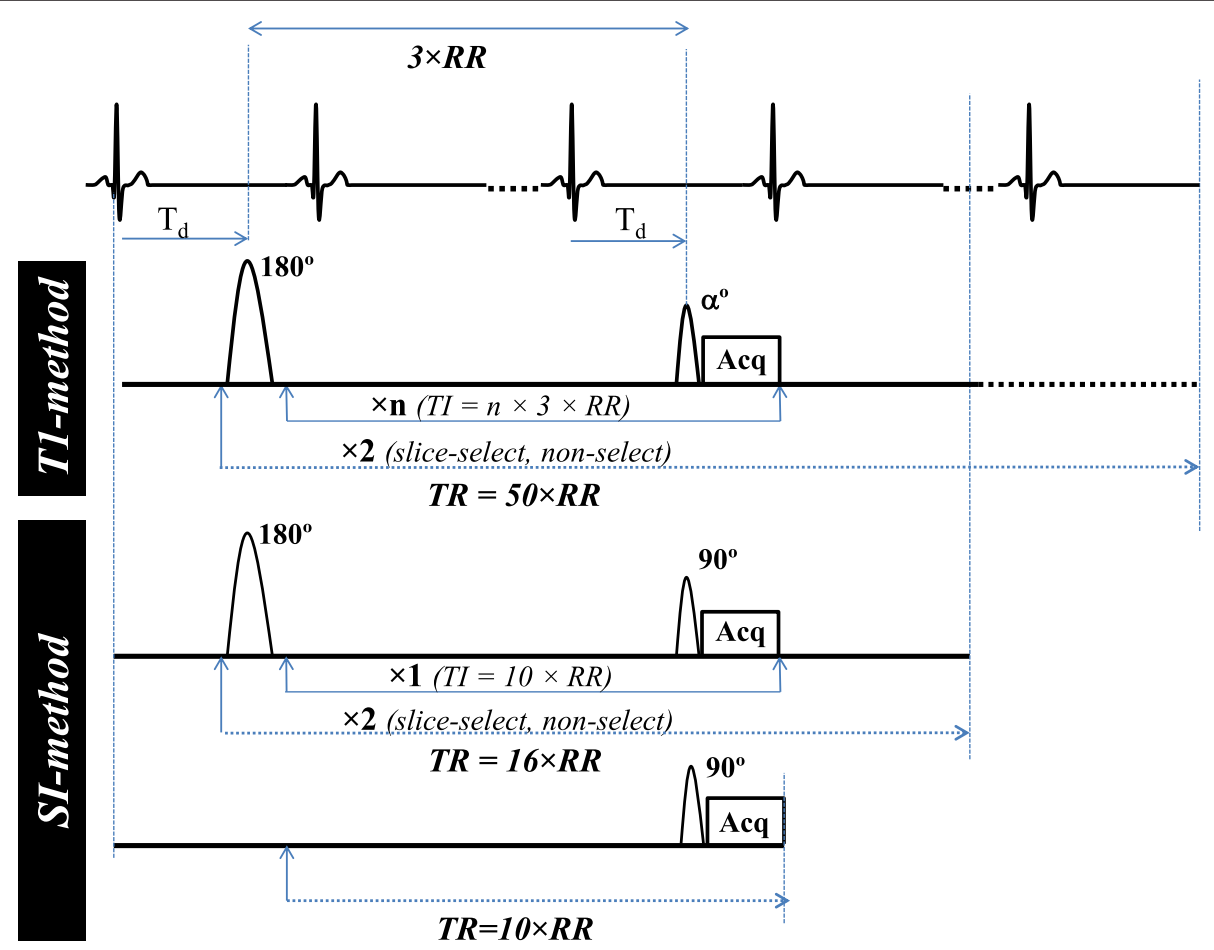

Figure 1 Acquisition schemes for the mouse cardiac studies. Slice select and non-select, segmented, Look-Locker inversion recovery sequences with data acquisitions at every third cardiac cycle was used for the conventional T1-method (top). The TR was set to 50 RR intervals in this case. The SI-method (bottom) consisted of three acquisitions: Slice select and non-select, segmented, inversion recovery sequences, each with one acquisition at 10XRR and a segmented gradient echo sequence with no spin preparation and TR set to 10XRR. In both methods the acquisition block comprised a single excitation pulse followed by one line of $k$ space. 
acquisition with TR set to TI. The method as it was implemented in mice is depicted in Figure 1. A segmented kspace acquisition is assumed. A finite TR is assumed for (i) and (ii). We will derive an expression for magnetization for each of these cases in terms of perfusion in a two compartment model of tissue (Figure 2) where we assume myocardial tissue to be consisting of homogenous intra and extra-capillary space with respect to relaxation time. The rate of change of magnetization in the intracapillary region $\mathrm{m}_{\mathrm{c}}(\mathrm{t})$ depends on loss of magnetization due to $T_{1}$ relaxation, exchange of magnetic spins between intra and extra-capillary region and gain of magnetization due to in flow of blood. The magnetization in the extra-capillary region $\mathrm{m}_{\mathrm{e}}(\mathrm{t})$ is unaffected by blood flow. The spin dynamics of the two compartments is described by the following modified Bloch equations.

$$
\begin{aligned}
\frac{d m_{c}(t)}{d t}= & \left(\frac{m_{c}(0)-m_{c}(t)}{T_{1 c}}\right)+\frac{F m_{p}}{V_{c}}-\frac{F m_{c}(t)}{V_{c}} \\
& -K_{c} m_{c}(t)+K_{e} m_{e}(t) \\
\frac{d m_{e}(t)}{d t}= & \left(\frac{m_{e}(0)-m_{e}(t)}{T_{1 e}}\right)+K_{e} m_{c}(t)-K_{e} m_{e}(t)
\end{aligned}
$$

Where $T_{l x}, m_{x}(t), V_{x}$ and $K_{x}$ are longitudinal relaxation time, magnetization, volume, spin exchange rate respectively with the subscript $x=c$ for intracapillary region and $x=e$ for extra-capillary region. $\mathrm{m}_{\mathrm{p}}(\mathrm{t})$ is the magnetization of the incoming blood. The total magnetization of tissue, $M(t)$, is calculated as,

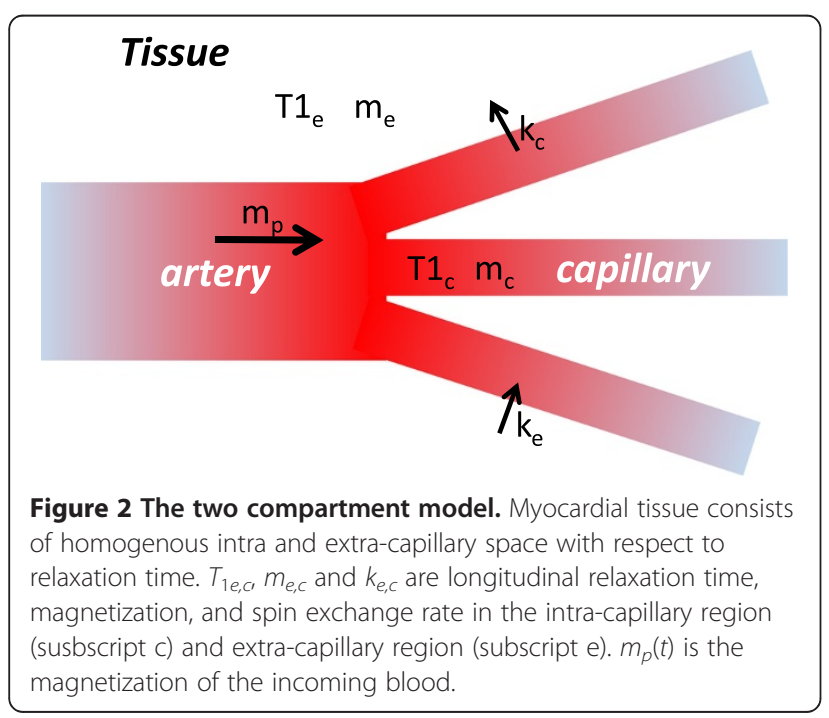

$$
M(t)=\left(\frac{R B V}{\lambda}\right) m_{c}(t)+\left(1-\frac{R B V}{\lambda}\right) m_{e}(t)
$$

where $\lambda$ is the blood tissue water partition coefficient [4], $R B V=\frac{V_{c}}{V_{t}}$ and $V_{T}=V_{c}+V_{e}$. Equations 1-3 describe the time evaluation of the total magnetization of tissue and can be solved for a given input magnetization, $\mathrm{m}_{\mathrm{p}}(\mathrm{t})$, depending on the type of spin preparation. For slice-select inversion, $\mathrm{m}_{\mathrm{p}}(\mathrm{t})$ is the equilibrium magnetization of capillary blood hence is equal to the constant $\mathrm{m}_{\mathrm{c}}(0)$ in magnitude. For non-select inversion, at steady state, $m_{p}(t)=m_{c}(0)\left(1-2 e^{-\frac{T I}{T_{1 c}}}\right)+m_{c}(0) e^{-\frac{T R}{T_{1 c}}}$, where $T I$ and $T R$ are the inversion and repetition times respectively (see Appendix).

The total magnetization for each spin preparation can be written in matrix from as follows:

$$
\frac{d}{d t}\left(M_{s}(t) e^{-R t}\right)=\frac{P}{R B V} e^{-R t} m_{c}(0)+e^{-R t}\left(\begin{array}{c}
\frac{m_{c}(0)}{T_{1 c}} \\
\frac{m_{e}(0)}{T_{1 e}}
\end{array}\right)
$$

for slice-select inversion and

$$
\begin{aligned}
\frac{d}{d t}\left(M_{g}(t) e^{-R t}\right)= & \frac{P}{R B V} m_{c}(0)\left(1-2 e^{-\left(\frac{T I}{T_{1 c}}\right)}+e^{-\left(\frac{T R}{T_{1 c}}\right)}\right) e^{-R t} \\
& +e^{-R t}\left(\begin{array}{c}
\frac{m_{c}(0)}{T_{1 c}} \\
\frac{m_{e}(0)}{T_{1 e}}
\end{array}\right)
\end{aligned}
$$

for non-select inversion, where

$$
R=\left[\begin{array}{cc}
-\left(\frac{1}{T_{1 c}}+\frac{F}{V_{c}}+K_{c}\right) & K_{c} \\
K_{e} & -\left(\frac{1}{T_{1 e}}+K_{e}\right)
\end{array}\right]
$$

Equations (5) and (6) can be solved to derive the magnetization for slice-select $\mathrm{M}_{\mathrm{s}}(\mathrm{t}=\mathrm{TI})$ and non-select inversion $\mathrm{M}_{\mathrm{g}}(\mathrm{t}=\mathrm{TI})$ using the initial condition that all magnetization within the selected slice is inverted at $\mathrm{t}=0$ in both preparation schemes. The term $R$ can be simplified following a series of steps proposed by Bauer et al. [1,12] assuming fast exchange between intra and extra capillary regions i.e. $\mathrm{Kc} \approx \mathrm{Ke} \approx \infty$. This assumption reduces the complexity of the model but is 
appropriate for myocardial tissue that has a high exchange rate compared to other tissues of the body [12]. The difference in the signal intensities between the two preparation schemes can now be defined as $\Delta M=M_{g}(t=T I)-M_{s}(t=T I)$. By combining the simplified expression for $R$ and the solutions to equations (5) and (6) we obtain,

$$
\Delta M=\frac{2 P m_{c}(0)\left(e^{-\left(\frac{T I}{T_{1 c}}\right)}-e^{-(\mu T T)}\right)}{\left(\mu-\frac{1}{T_{\mathrm{lc}}}\right) \lambda}+\frac{P m_{c}(0) e^{-\left(\frac{T R}{T_{\mathrm{Ic}}}\right)}\left(e^{-(\mu T T)}-1\right)}{\mu \lambda}
$$

Where, $\mu=\left(\frac{R B V}{\lambda}\right) \frac{1}{T_{1 c}}+\left(1-\frac{R B V}{\lambda}\right) \frac{1}{T_{1 e}}+\frac{P}{\lambda}$

To remove the dependency on receiver characteristics, we normalize $\Delta \mathrm{M}$ by the magnetization of a steady state gradient echo acquisition $\left(\mathrm{M}_{\mathrm{s}}\right)$ with no spin preparation and with $T R$ set to the same $T I$ as in equation (7) (Figure 1). Since echo time of this acquisition is small ( $\sim 1 \mathrm{~ms})$ we assume that $M_{s}$ is not affected by perfusion. We also assume equilibrium magnetization of intra and extra-capillary regions to be similar i.e. $m_{c}(0) \approx m_{e}(0)$. Thus we obtain,

$$
M_{s}=m_{c}(0)\left(1-e^{-\mu_{0} T I}\right)
$$

Where, $\mu_{0}=\left(\frac{R B V}{\lambda}\right) \frac{1}{T_{1 c}}+\left(1-\frac{R B V}{\lambda}\right) \frac{1}{T_{1 e}}=\frac{1}{T_{1}}$

Here $T_{1}$ is the myocardial longitudinal relaxation time without the in-flow effects. By dividing equation (7) by (8) we obtain an expression for the normalized magnetization difference, $\Delta M / M_{s}$ Then by taking the first order Taylor expansion of $\Delta \mathrm{M} / \mathrm{M}_{\mathrm{s}}$ about $T I$ we obtain,

$$
P=\frac{\frac{\lambda \Delta M}{M_{s}}}{\left(1-e^{-\frac{T R}{T_{1 c}}+e^{-\frac{T I}{T_{1 c}}}}\right) T_{1}}
$$

\section{SI-method for a non-exchangeable two compartment model of tissue}

Perfusion is derived for a non-exchangeable model of two compartments based on the same acquisition method as described above. The aim is to test the SI-method on a flow phantom. Since water exchange rates of tubing material is difficult to assess we chose material with zero permeability. This allows perfusion to be known exactly in terms of volume of fluid / volume of phantom / minute (i. e. $\min ^{-1}$ ), given a specific flow rate. The phantom will have two compartments, intra- and extra capillary, but with no exchange between them. Accordingly the SI-method is modified for this scenario by making $\mathrm{K}_{\mathrm{c}}=0, \mathrm{~K}_{\mathrm{e}}=0$ in equations (2) and (3). The rest of the derivation is similar to as in the previous section.

The difference in the signal intensities between the two preparation schemes, $\Delta \mathrm{M}$, is obtained by first order Taylor expansion as in the previous section. By solving for perfusion we get,

$$
P=\frac{\lambda \Delta M}{m_{c}(0)\left(2 e^{-\frac{T I}{T_{1 c}}}-e^{-\frac{T R}{T_{1 c}}}\right) T I}
$$

Here we do not normalize $\Delta M$ by the signal intensity of a steady state acquisition, instead measure $m_{c}(0)$, the equilibrium magnetization of the capillary fluid, using a spin echo sequence with long TR.

\section{T1-method for a non-exchangeable two compartment model of tissue}

The aim is to compare the results of the T1-method with that of the SI-method using the flow phantom described above. The T1-method was originally developed by Bauer et al. [4] for a fast exchanging two compartment model of tissue. Here we modify it to reflect no exchange between compartments. Again we begin with setting $\mathrm{K}_{\mathrm{c}}=0, \mathrm{~K}_{\mathrm{e}}=0$ in equations (2) and (3). In the T1-method we are interested in the apparent longitudinal relaxation times and not the magnitudes of magnetization. To make the derivation of relaxation times easier we normalize the magnetization of intra and extra-capillary regions at the time of inversion to one. The equilibrium magnetization on the other hand is set to zero i.e. $m_{c}(0)=m_{e}(0)=0$ in equations (2) and (3). The total magnetization of tissue, $M(t)$, is given by equation (4) as before. Modified Equations 2-4 can be solved for a given input magnetization, $\mathrm{m}_{\mathrm{p}}(\mathrm{t})$, depending on the type of spin preparation. According to the normalizing scheme introduced here the input magnetization, $\mathrm{m}_{\mathrm{p}}(\mathrm{t})$, for slice-select inversion is zero and $m_{p}(t)=e^{-\frac{t}{T_{1 c}}}$, for non-select inversion.

By taking into account the mean relaxation time approximation that $T_{1}=\int{ }_{0}^{\infty} \mathrm{M}(t) d t,[4,13]$, we obtain the following expressions for apparent relaxation times

$$
T_{1 s}=\frac{R B V}{\lambda}\left(\frac{R B V T_{1 c}}{P T_{1 c}+R B V}\right)+\left(1-\frac{R B V}{\lambda}\right) T_{1 e}
$$

for slice-select inversion, and

$$
T_{1 g}=\frac{R B V}{\lambda} T_{1 c}+\left(1-\frac{R B V}{\lambda}\right) T_{1 e}
$$

for non-select inversion. From equations (11) and (12) we derive an expression for perfusion in a two compartment model of tissue with no exchange.

$$
P=\frac{1}{T_{1 c}\left\{\frac{1}{\lambda}\left(\frac{T_{1 c}}{T_{1 g}-T_{1 s}}\right)-\frac{1}{R B V}\right\}}
$$

The $R B V$ in the context of the said phantom is equal to the volume ratio $\mathrm{V}_{\mathrm{c}} / \mathrm{V}_{\mathrm{T}}$, where $V_{c}$ is the volume of capillary tube and $V_{T}$ is the total volume 
of the phantom as measured from a cross section of the phantom.

\section{Two compartmented flow phantom study}

A phantom was prepared by placing 12 loops of capillary tube (Cole Parmer \# EW 06492-02) with inner diameter $0.51 \mathrm{~mm}$, inside a $3 \mathrm{cc}$ syringe. One end of the capillary tube was fitted to a syringe infusion pump (Model R99E, Razel Scientific Instruments, St. Albans, VT). The space between capillary tubes was filed with a mixture of Gadolinium and Poly Vinyl Alcohol solution. Diluted Gd solution was used as the capillary liquid. Intra and extra-capillary liquids were prepared to have approximately similar longitudinal relaxation times of blood and tissue at 7 Tesla $\left(T_{1 \text {-intra }}=1896 \mathrm{~ms}, T_{1-\text { extra }}=\right.$ $1344 \mathrm{~ms})$. Perfusion was calculated using equation (10) for the SI-method and equation (13) for the T1-methods for the following flow velocities $0.03,0.07,0.09,0.1,0.18,0.3$, $0.35 \mathrm{~cm} \mathrm{~s}^{-1}$. The average signal intensity of a region covering the phantom was considered for calculation of $\Delta M$ and for curve fitting of $T_{1}$.

Cardiovascular magnetic resonance (CMR) was performed on a 7T Bruker Biospec system using an inversion recovery FLASH sequence consisting of a sinc3 excitation pulse and a hyperbolic secant pulse (sech) for inversion. For the T1-method, slice select inversion and non-select inversion prepared images were acquired at a constant repetition time, varying the inversion time, TI, in twelve steps between 50 and $5500 \mathrm{~ms}$. TR/TE $=6000$ $\mathrm{ms} / 2.6 \mathrm{~ms}$ field of view $=2.5 \times 2.5 \mathrm{~cm}^{2}$, flip angle $=30^{\circ}$, slice thickness $=4 \mathrm{~mm}$, a matrix size $=256 \times 64$. For the SI-method, TR $=1000 \mathrm{~ms}$ and $T I=100 \mathrm{~ms}$, flip angle $=90^{\circ}$, All other imaging parameters were set similar to that of the T1-method. Imaging was performed perpendicular to the direction of flow. For both methods, the average signal intensity of a region of interest covering a cross section of the phantom was considered for calculations. $T_{1 s}$ and $T_{1 g}$ were calculated by fitting to a mono exponential function of the form $A e^{-\frac{T I}{T_{1}}}+B$. Spin density of intra $\left(\rho_{\mathrm{i}}\right)$ and extra-capillary $\left(\rho_{\mathrm{e}}\right)$ liquids were obtained by a spin echo pulse sequence with $T R=20 \mathrm{sec}$ and with all other imaging parameters including receiver gains fixed. Perfusion was calculated using equation (10) for SI-method and equation (13) for T1-method taking $\lambda=\left(\rho_{i}+\rho_{e}\right) / \rho_{i}$ and $m_{c}(0)=\rho_{i}$.

\section{In vivo mouse study}

The study was conducted under a protocol approved by the Institutional Animal Care and Use Committee. To compare the proposed SI-method with the conventional T1-method, we measured myocardial perfusion of healthy ten weeks old C57BL/6 mice $(n=12)$ using both methods. Mice were anesthetized with continuous inhaled Isoflurane (2\% by volume) administered via a nose cone. Constant body temperature of $37^{\circ} \mathrm{C}$ was maintained using a thermocouple/heater system. Both SI and T1 methods were performed in the same session. To test the reproducibility of the SI-method three perfusion measurements were made on the same mouse. Each measurement was performed on a separate session (often on a different day). Three C57BL/6 mice were used for this study. Finally we used the SI-method to quantify perfusion in a mouse model of ischemiareperfusion (IR). Four C57BL/6 mice were anesthetized with isoflurane and intubated through the mouth and ventilated. The left coronary artery was ligated with a silk ligature following thoracotomy. The animals were maintained in the ligated state for 30 minutes, after which the externalized silk was pulled to release the constriction and hence allowing full reperfusion. IR mice were scanned within 24 hours of surgery. The in vivo study was performed on a $7 \mathrm{~T}$ Bruker Biospec system using a custom made solenoid coil having a single turn consisting of a wide conductive sheet (diameter $=3.0 \mathrm{~cm}$, length $=3.5 \mathrm{~cm}$ ). The sensitivity of the coil covered approximately $80 \%-90 \%$ of the mouse's body. In IR mice, in addition to myocardial perfusion measurement, LGE experiment was performed 30 minutes after injecting a bolus of Gd-DTPA $(0.3-0.6 \mathrm{mmol} / \mathrm{kg})$ intraperitonealy. $T 1$ weighted cine imaging was performed in the short axis using a segmented FLASH sequence to highlight enhancement. Slice thickness $=1.0 \mathrm{~mm}$, matrix size $=256 \times 256$, in-plane resolution $=117 \times 117 \mu \mathrm{m} 2$. TE/TR $=3 / 5.2 \mathrm{~ms}$, flip angle $=30^{\circ}$. Image acquisition was prospectively ECG gated using pediatric ECG probes attached to the paws. A pneumatic pillow was used for respiratory gating (SA Instruments, Stony Brooks, NY).

The acquisition schemes for T1 and SI methods are shown in Figure 1. A gated, Look-Locker sequence [5] was implemented for the T1-method. A 5ms hyperbolic secant pulse was used for inversion. For the T1-method, for each inversion, the relaxation curve was sampled at twelve different inversion times at every third cardiac cycle i.e. $T I=3 \times n \times R R, n=1, . .12$ with $R \approx 50 \times R R$, that is approximately 6 seconds. For the SI-method the same sequence was used with data acquired at a single $T I$ time of $10 \times R R$ with $T R=16 \times R R$, that is approximately 2 seconds. In both methods the acquisition block comprised a single excitation pulse followed by one line of $\mathrm{k}$ space. For the SI-method an additional steady state gradient echo image was acquired without the inversion pulse with $\mathrm{TR}=10 \times \mathrm{RR}$. All images were acquired in the short axis plane at end diastole. The following common imaging parameters were used: $\mathrm{TE}=1.73 \mathrm{~ms}$, field of view $=2.5 \times 2.5 \mathrm{~cm}^{2}$, slice thickness $=2.0 \mathrm{~mm}$, matrix size $=128 \times 64$, flip angle $=90^{\circ}$ and $10^{\circ}$ were used for SI and T1 method respectively. For slice select 
inversion, the slice thickness of the inversion pulse was set to twice that of the excitation slice to eliminate effects from imperfect edges of both the inversion and the imaging pulse profiles

For the in vivo study we assumed myocardial tissue to consist of fast exchanging two compartments. Therefore we used equation (9) for the SI-method, and equation (1) for the T1-method for calculating perfusion. Following constant values were used: $\mathrm{T}_{1}$ of blood, $\mathrm{T}_{1 \mathrm{c}}=1800 \mathrm{~ms}$, $\mathrm{T}_{1}$ of myocardial tissue in the absence of flow effects, $\mathrm{T}_{1}=1400 \mathrm{~ms}, \lambda=0.95 \mathrm{ml} / \mathrm{g}$ [14]. Perfusion was calculated on a pixel-by-pixel basis. The mean value was calculated from an ROI drawn within the left ventricle wall. Pixel values less than $-5 \mathrm{ml} / \mathrm{g} / \mathrm{min}$ and greater than $15 \mathrm{ml} / \mathrm{g} / \mathrm{min}$ were set to zero and also threshold limit was set to obtain a noise free perfusion map. The apparent $T_{1}$ values obtained by the Look Locker acquisition was corrected for saturation effects as previously described [5].

Results are expressed as mean \pm standard deviation. Statistical differences were assessed with the unpaired 2-tailed Student's t test for two experimental groups. A nonparametric test was applied when the data were not normally distributed. A 2-tailed $\mathrm{P}$ value of less than 0.05 was considered statistically significant.

\section{Results}

The results of the phantom experiments are shown in Figure 3. In this case the perfusion is expressed as the flow rate per unit volume of phantom i.e. in $(\mathrm{ml} / \mathrm{min})$ / (ml). Perfusion measured by both methods agrees well with that calculated using the flow rates in the capillary tubes. The deviation from the actual perfusion is greater for higher flow rates for both methods, the maximum being $16 \%$. The Bland Altman plots show that the difference between the executed perfusion and the measured stay within the $95 \%$ confidence level in both cases.

The error bars shown in Figure 3 are derived by propagating errors of $P$ in equations (10) and (13) for SI and $T 1$ methods. Larger errors in the T1-method reflect greater uncertainty of $T_{1 s}$ and $T_{1 g}$ measurements.

Typical left ventricular perfusion maps of a normal mouse, by SI and T1-method are given in Figure 4. The average $T_{1 s}$ and $T_{1 g}$ for normal mice in our study was $1293 \pm 80 \mathrm{~ms}$ and $1542 \pm 88 \mathrm{~ms}$ respectively. The Table 1 compares the perfusion values obtained by the two methods for each mouse. Perfusion was calculated from region of interest covering the entire left ventricle wall. The myocardial perfusion of healthy mice obtained by the SI-method is $5.6 \pm 0.5 \mathrm{ml} / \mathrm{g} / \mathrm{min}$, (mean \pm standard deviation). This is not significantly different $(\mathrm{p}>0.05)$ to that obtained by the T1 method, $5.6 \pm 0.3 \mathrm{ml} / \mathrm{g} / \mathrm{min}$. The standard deviation given for each mouse in Table 1 reflects the variation of $P$ within the left ventricle for each mouse. The mean value of this standard deviation is significantly higher for the T1-method $(2.3 \mathrm{ml} / \mathrm{g} / \mathrm{min})$ compared to that of the SI-method $(1.6 \mathrm{ml} / \mathrm{g} / \mathrm{min})$, $\mathrm{p}<0.0001$. As seen in Table 2 the mean percentage standard deviation among repeated measures is less than $5 \%$ for the SI-method.
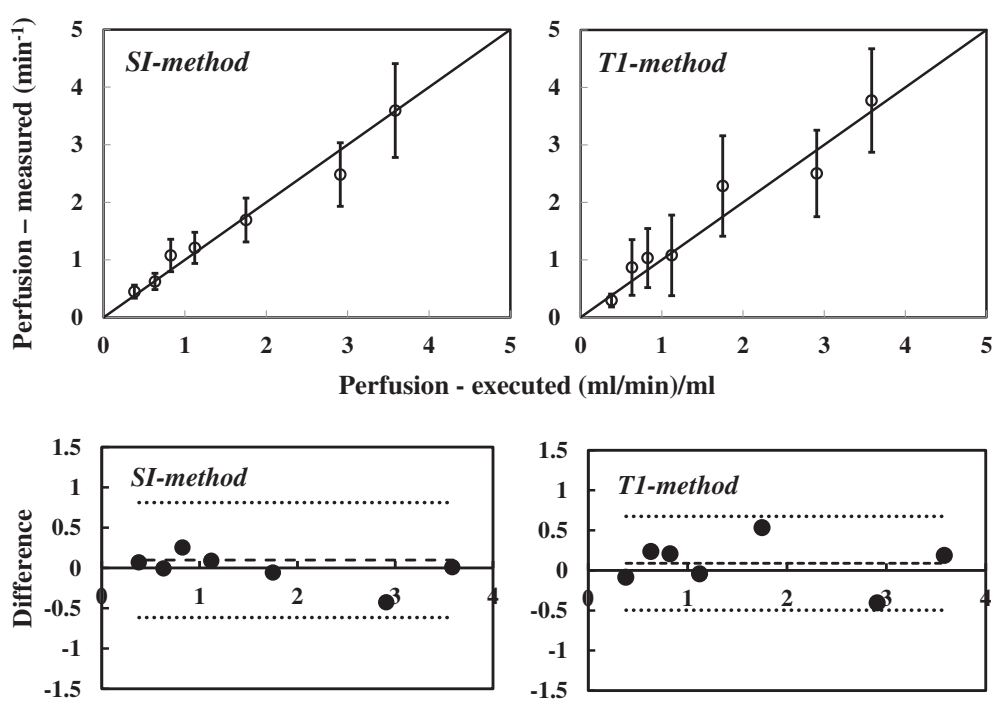

Perfusion - executed $(\mathrm{ml} / \mathrm{min}) / \mathrm{ml}$

Figure 3 Comparison of phantom results. The perfusion measured by SI- (left) and T1-methods (right) is compared with that calculated from the flow rates. The Bland Altman plots show that the difference between the executed perfusion and the measured are within the $95 \%$ confidence level in both cases. 

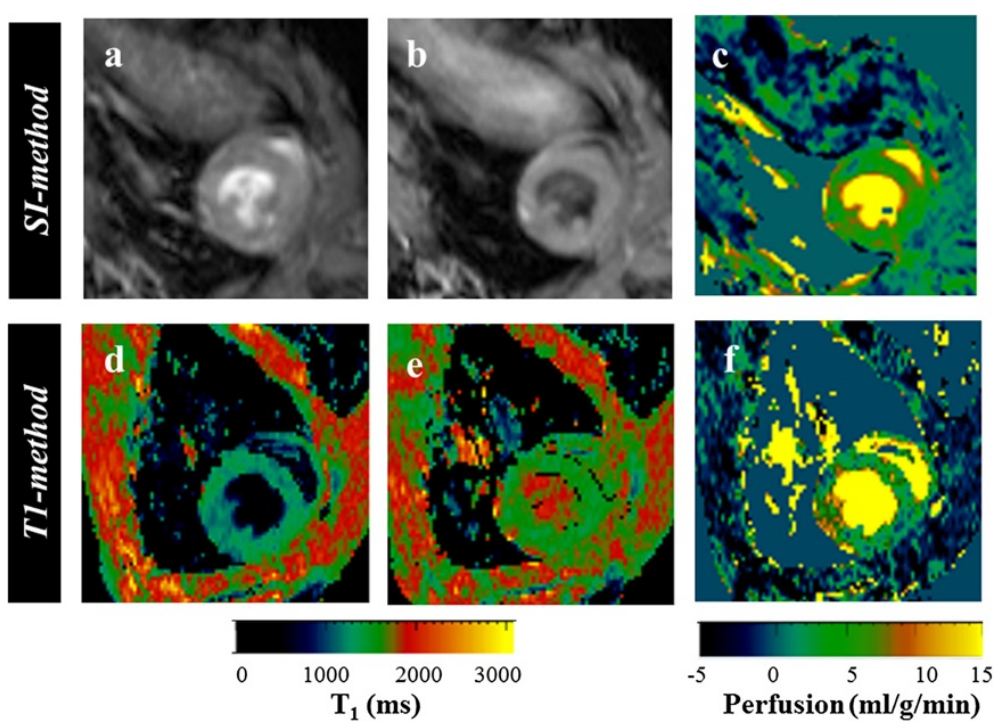

Figure 4 Comparison of in vivo perfusion maps. Example of raw images and calculated perfusion maps of the left ventricle in short axis plane are shown for each technique. SI-method: (a) slice-select inversion image, (b) non-select inversion image, (c) perfusion. T1-method: (d) $T_{1 s}$ map, (e) $T_{1 g}$ map, (f) perfusion.

Figure 5 shows an example of LGE and perfusion in an IR mouse in the short axis plane. The enhancement in the lateral wall and the anterior septum are matched with regions of hypo-perfusion in the perfusion map. The average perfusion in the hypoperfused region among all four IR mice was $1.2 \pm 0.9 \mathrm{ml} / \mathrm{g} / \mathrm{min}$ and that of the remote region was $4.4 \pm 1.2 \mathrm{ml} / \mathrm{g} / \mathrm{min}$.

\section{Discussion}

In this study, a new ASL method to quantify myocardial perfusion is introduced based on the signal intensity of

Table 1 Comparison of in vivo results for each mouse

\begin{tabular}{lccccc}
\hline Mouse ID & \multicolumn{2}{c}{ SI-method $(\mathbf{m l} / \mathbf{g} / \mathbf{m i n})$} & & \multicolumn{2}{c}{ T1-method $\mathbf{( m l} / \mathbf{g} / \mathbf{m i n})$} \\
\cline { 2 - 3 } \cline { 5 - 6 } & Mean & $\begin{array}{c}\text { Standard } \\
\text { deviation }\end{array}$ & & Mean & $\begin{array}{c}\text { Standard } \\
\text { deviation }\end{array}$ \\
\hline 1 & 5.8 & 2.0 & & 5.6 & 2.9 \\
2 & 5.6 & 1.8 & 5.7 & 2.7 \\
3 & 5.7 & 1.8 & & 5.4 & 2.1 \\
4 & 6.4 & 1.9 & & 6.1 & 2.2 \\
5 & 6.2 & 1.8 & & 5.5 & 2.9 \\
6 & 4.8 & 1.3 & & 5.1 & 2.1 \\
7 & 5.5 & 1.8 & & 5.6 & 2.0 \\
8 & 5.9 & 1.6 & & 6.1 & 2.2 \\
9 & 5.7 & 1.8 & & 5.9 & 2.5 \\
10 & 5.3 & 1.3 & & 5.3 & 2.1 \\
11 & 5.8 & 1.7 & & 5.6 & 2.5 \\
12 & 4.8 & 1.3 & & 5.0 & 2.2 \\
Average & $\mathbf{5 . 6} \pm \mathbf{0 . 5}$ & $\mathbf{1 . 6}$ & & $\mathbf{5 . 6 \pm 0 . 3}$ & $\mathbf{2 . 3}$ \\
\hline
\end{tabular}

flow sensitized CMR. Following Bauer's [1] frame work, myocardium is modeled as a two compartment with respect to $T_{1}$, which takes in to consideration that $T_{1}$ of capillary blood is different to that of tissue. The imaging method consists of segmented, single gradient echo acquisitions prepared by slice select and non-select inversion pulses with finite repetition times followed by a gradient echo acquisition with no spin preparation. A simple expression for perfusion was derived by solving a modified Bloch equation for this model.

An important feature of our tissue model is that it consists of two compartments. In contrast, the Detre's frame work [15] and its derivative ASL methods by Kwong [9], Buxton [8] and Zun [10] considers the tissue to be consists of a single compartment of blood. However, because $T_{1}$ of blood is higher than that of tissue, taking tissue to be a single compartment of blood could lead to overestimation of perfusion. This is problematic if absolute values of perfusion are desired. On the other hand, we can arrive at the same expression for $P$ as did Kwong and others by reducing our model to a single compartment model. To achieve this we take the two compartment non-exchange version of the SI-method

Table 2 Reproducibility of the SI-method

\begin{tabular}{|c|c|c|c|c|c|}
\hline \multirow{2}{*}{$\begin{array}{l}\text { Mouse } \\
\text { ID }\end{array}$} & \multicolumn{3}{|c|}{ Perfusion $(\mathrm{ml} / \mathrm{g} / \mathrm{min})$} & \multirow[b]{2}{*}{$\begin{array}{l}\text { Mean } \pm \text { std. } \\
\text { dev }\end{array}$} & \multirow[t]{2}{*}{ (std. dev/mean)*100 } \\
\hline & exp. 1 & exp. 2 & exp. 3 & & \\
\hline 1 & 4.8 & 5.0 & 5.2 & $5.0 \pm 0.2$ & $4 \%$ \\
\hline 2 & 5.3 & 5.8 & 5.7 & $5.6 \pm 0.2$ & $4.8 \%$ \\
\hline 3 & 6.2 & 6.3 & 6.4 & $6.3 \pm 0.1$ & $2 \%$ \\
\hline
\end{tabular}




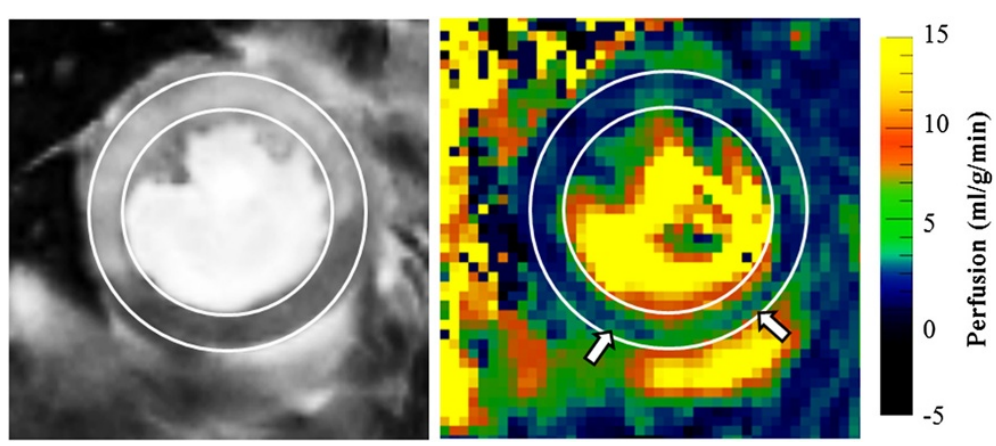

Figure 5 Perfusion in the ischemia-reperfusion model. LGE in the lateral wall and the anterior septum (left) are matched with regions of hypo-perfusion in the perfusion map (right). Arrows show low perfusion regions extending to the mid-myocardium beyond the boundary of the enhancement.

(Equation 10) which is similar to one compartment of blood as far as $\Delta M$ is concerned. Then, by making $T R \rightarrow \infty$ in equation 10 we obtain the same expression for $P$ as in equation 5 of reference 9 . Because of the two compartment model, the SI-method requires the knowledge of $T_{1}$ of tissue with no in-flow effects $\left(T_{1}\right)$ (Equation (9)) in addition to the $T_{1}$ of blood $\left(T_{1 c}\right)$ whereas Detre's method only requires the knowledge of $T_{1 c}$. In this paper we have presented $P$ calculated using literature values of $T_{1}$ and $T_{1 c}$. However, in our case these relaxation times could also be calculated from data acquired for the T1-method. Hence we calculated $T_{1}$ and $T_{1 c}$ for each mouse in Table 1 and used it to derive $P$ using the SI-method (data not shown). The average $P$ calculated using individually assessed $T_{1}$ and $T_{1 c}$ was $5.7 \pm 0.4 \mathrm{ml} / \mathrm{g} / \mathrm{min}$. Since this is not significantly different to the average $P$ calculated using literature values $(=5.6 \pm 0.5 \mathrm{ml} / \mathrm{g} / \mathrm{min})$ it appears that the variability of perfusion among mice is greater than the uncertainty posed by $T_{1}$ and $T_{1 c}$ in the SI-method.

The other important feature of the SI-method is that its k-space acquisition scheme is segmented as opposed to a single shot acquisition. Also, in deriving $P$ no assumption was made about the length of the repetition time. This allows phase encoding steps to be acquired in multiple inversions with shorter TR times than it is required for full relaxation. Unlike in humans, the cardiac cycle in small animals is too short $(\sim 150 \mathrm{~ms}$ under anesthesia) to acquire a full set of k-space data in a single inversion. Therefore, by allowing for short repetition times, the SI-method considerably shortens the total acquisition time for high resolution experiments. Depending on the heart rate, the total scan time for the T1-method ranged from 6-8 minutes while that for the SI-method was 2-4 minutes. In human studies, the single shot acquisition limits the in-plane resolution since all k-space data must be acquired in a short period of time in diastole. In this context the segmented acquisition scheme of the SImethod and its theoretical model allows to achieve high resolution perfusion maps if the temporal noise could be mitigated.

The phantom and the in vivo study results show that the $P$ values measured with the SI-method is comparable with that measured with the conventional T1-method. With the flow phantom we were able to verify the accuracy of the SI-method albeit with no spin exchange effects. In order to apply the SI-method to the flow phantom, the theoretical model was modified to reflect spin non-exchangeability. In this respect, the phantom is an exact physical replica of the modified theoretical model. Therefore the accuracy of the predicted outcome carried no uncertainty due to the design of the model. Hence the phantom experiment was an ideal method to test the relationship of $P$ to flow sensitized MR signal as predicted by physics. However, in reality it is difficult to ascertain how well the two compartment model mimic myocardial tissue.

The fluorescent microspheres or fluospheres, have recently become a popular alternative to radioactive microspheres for quantitating myocardial perfusion in rodent experimental studies. The myocardial perfusion of healthy C57BL/6 mice measured using fluorescent microspheres $(5.7 \pm 0.3 \mathrm{ml} / \mathrm{g} / \mathrm{min})$ by Richer et al. [16] closely matches that of the SI-method $(5.6 \pm 0.4 \mathrm{ml} / \mathrm{g} / \mathrm{min})$ and the $T 1$ method $(5.6 \pm 0.3 \mathrm{ml} / \mathrm{g} / \mathrm{min})$ in our study. As seen in

Table 3 Published values of myocardial perfusion of healthy mice using CMR methods

\begin{tabular}{ll}
\hline Reference & Perfusion (ml/g/min) \\
\hline [18] Makowski M. et al., 2010 & $7.3 \pm 0.8^{*}$ \\
[6] Streif JU. et al., 2005 & $7.0 \pm 0.5^{*}$ \\
[19] Nahrendorf M. et al., 2006 & $6.7 \pm 0.3^{*}$ \\
[14] Kober F. et al., 2005 & $6.0 \pm 1.9^{+}$ \\
Proposed SI-method & $\mathbf{5 . 6} \pm \mathbf{0 . 5} \mathbf{+}^{+}$ \\
[17] Vandsburger MH. et al., 2010 & $5.2 \pm 0.8^{*}$ \\
[20] Vandsburger MH. et al., 2007 & $4.3 \pm 0.3^{*}$ \\
\hline
\end{tabular}

" standard error, ${ }^{+}$standard deviation. 
Table 3, our values are also within the range of published values $[6,14,17-20]$, for myocardial perfusion in mice using standard ASL methods. The mean standard deviation of repeated measures by the SI-method is $3.6 \%$. This shows that the perfusion quantified using the proposed method is highly reproducible. Interestingly we noted that the variation of $P$ within the myocardium was significantly higher for the T1-method compared to that of the SI-method in the in vivo study. A probable cause is the variation in beat to beat time difference in the cardiac cycle resulting errors in the inversion times. This problem is exuberated by the long scan time of the T1-method. However, recently introduced post processing techniques could be used to mitigate this error [17].

In general, regions of LGE matched with regions of hypo-perfusion in the ischemia reperfusion study. But in some cases, low perfusion regions extended to the midmyocardium beyond the boundary of the enhancement (arrows, Figure 3). It is possible that contrast material was not retained in these areas since it was surrounded by normally perfused tissue. It should also be noted that the perfusion maps have a lower resolution than that of the LGE images. Therefore these low perfusion extensions represents some volume averaging.

Although the slice thickness of the inversion pulse was twice that of the excitation pulse we believe it was much less in the actual implementation since the RF pulse shapes were significantly different. The goal was to compensate for miss alignment of the inversion and excitation slice profiles due to motion. However the thicker inversion slice underestimates perfusion. The shorter scan time of the SI-method somewhat reduces the errors due to motion thereby allowing for better alignment of the two profiles. This error can be vastly mitigated in human studies where single shot acquisitions can be used. Recently published work by Zun et al. demonstrates the feasibility of such a signal based ASL method with a single shot acquisition in humans [10].

The aim here was to introduce a new theoretical frame work for signal based ASL but we admit that much work remains to be done to improve the imaging technique. The quality of the inversion is problematic for high fields in general where B1 field inhomogeneity is much worse; thus remains a source of error in this application. Transit time effects and coil inflow time effects are associated with improper inversion pulses. These issues were not addressed in this study. We refer the readers to a rigorous analysis of these effects in the context of short TR by Pell et al. [11]. Compared to brain studies physiological noise is another major factor affecting the quality of cardiac perfusion maps. Some tasks for future consideration are: reducing physiological noise, overcoming mis-registration of differently prepared images, achieving a better slice profile for inversion, correcting for varied
TI times of different k-space lines. With these improvements in place, the SI-method could potentially be used to generate high resolution pixel-by-pixel perfusion maps in human hearts.

\section{Conclusion}

This study demonstrates that signal intensity based ASL method is a robust alternative to the conventional T1method. The proposed SI-method with a segmented acquisition scheme allows faster high resolution perfusion mapping in small animals. Compared to other signal intensity based ASL methods, the two compartmented model used in the present study makes it biologically more accurate.

\section{Appendix}

During repeated inversion pulses at intervals of TR the longitudinal magnetization relaxes as,

$$
M_{z}(T I)=M_{z}(0) e^{-\frac{T I}{T_{1}}}+M_{0}\left(1-e^{-\frac{T I}{T_{1}}}\right)
$$

The $90^{\circ}$ excitation pulse saturates the $M_{z}$ and subsequently recovers during the time TR-TI to

$$
M_{n}^{-}=M_{0}\left(1-e^{-\frac{T R-T I}{T_{1}}}\right)
$$

just before the nth inversion pulse. Therefore just after the nth inversion pulse the magnetization is given by;

$$
M_{n}^{+}=-M_{n}^{-}
$$

Combining (1) (2) and (3) we get,

$$
M_{z}(T I)=-M_{0}\left(1-e^{-\frac{T R-T I}{T_{1}}}\right) e^{-\frac{T I}{T_{1}}}+M_{0}\left(1-e^{-\frac{T I}{T_{1}}}\right)
$$

and

$$
M_{Z}(T I)=M_{0}\left(1-2 e^{-\frac{T I}{T 1}}+e^{-\frac{T R}{T_{1}}}\right)
$$

\section{Competing interests}

The authors declare that they have no competing interests.

\section{Authors' contributions}

SA: Contributed to the design, mathematical derivations, acquisition and analysis of data, and drafting the manuscript; MS: Performed ischemia reperfusion surgery. JPW: Contributed to the conception and design, supervised acquisition, analysis and interpretation of data, involved in drafting the manuscript. All authors read and approved the final manuscript.

\section{Acknowledgements}

This work was supported by the NIH Heart, Lung and Blood Institute grant K25HL102244. 


\section{Author details}

${ }^{1}$ Department of Physics, University of Cincinnati, Cincinnati, OH, USA. ${ }^{2}$ Imaging Research Center, Department of Radiology, Cincinnati Children's Hospital, 3333 Burnet Ave, MLC 5033, Cincinnati, OH, 45229, USA. ${ }^{3}$ Howard Hughes Medical Institute, Molecular Cardiovascular Biology, Cincinnati Children Hospital, Cincinnati, OH, USA.

Received: 16 May 2012 Accepted: 11 October 2012

Published: 24 October 2012

\section{References}

1. Bauer WR, Hiller KH, Roder F, Rommel E, Ertl G, Haase A: Magnetization exchange in capillaries by microcirculation affects diffusion-controlled spin-relaxation: a model which describes the effect of perfusion on relaxation enhancement by intravascular contrast agents. Magn Reson Med 1996, 35(1):43-55.

2. Northrup BE, McCommis KS, Zhang H, Ray S, Woodard PK, Gropler RJ, Zheng J: Resting myocardial perfusion quantification with CMR arterial spin labeling at 1.5 T and 3.0 T. J Cardiovasc Magn Reson 2008, 10(1):53.

3. Wacker CM, Fidler F, Dueren C, Hirn S, Jakob PM, Ertl G, Haase A, Bauer WR Quantitative assessment of myocardial perfusion with a spin-labeling technique: preliminary results in patients with coronary artery disease. J Magn Reson Imaging 2003, 18(5):555-560.

4. Belle V, Kahler E, Waller C, Rommel E, Voll S, Hiller KH, Bauer WR, Haase A: In vivo quantitative mapping of cardiac perfusion in rats using a noninvasive MR spin-labeling method. J Magn Reson Imaging 1998, 8(6):1240-1245.

5. Kober F, Iltis I, Izquierdo M, Desrois M, Ibarrola D, Cozzone PJ, Bernard M: High-resolution myocardial perfusion mapping in small animals in vivo by spin-labeling gradient-echo imaging. Magn Reson Med 2004, 51(1):62-67.

6. Streif JU, Nahrendorf M, Hiller KH, Waller C, Wiesmann F, Rommel E, Haase A, Bauer WR: In vivo assessment of absolute perfusion and intracapillary blood volume in the murine myocardium by spin labeling magnetic resonance imaging. Magn Reson Med 2005, 53(3):584-592.

7. Zhang H, Shea SM, Park V, Li D, Woodard PK, Gropler RJ, Zheng J: Accurate myocardial T1 measurements: toward quantification of myocardial blood flow with arterial spin labeling. Magn Reson Med 2005, 53(5):1135-1142.

8. Buxton RB, Frank LR, Wong EC, Siewert B, Warach S, Edelman RR: A general kinetic model for quantitative perfusion imaging with arterial spin labeling. Magn Reson Med 1998, 40(3):383-396.

9. Kwong KK, Chesler DA, Weisskoff RM, Donahue KM, Davis TL, Ostergaard L, Campbell TA, Rosen BR: MR perfusion studies with T1-weighted echo planar imaging. Magn Reson Med 1995, 34(6):878-887.

10. Zun Z, Wong EC, Nayak KS: Assessment of myocardial blood flow (MBF) in humans using arterial spin labeling (ASL): feasibility and noise analysis. Magn Reson Med 2009, 62(4):975-983.

11. Pell GS, Thomas DL, Lythgoe MF, Calamante F, Howseman AM, Gadian DG Ordidge RJ: Implementation of quantitative FAIR perfusion imaging with a short repetition time in time-course studies. Magn Reson Med 1999, 41(4):829-840.

12. Bauer WR, Roder F, Hiller $\mathrm{KH}$, Han H, Frohlich S, Rommel E, Haase A, Ertl G: The effect of perfusion on T1 after slice-selective spin inversion in the isolated cardioplegic rat heart: measurement of a lower bound of intracapillary-extravascular water proton exchange rate. Magn Reson Med 1997, 38(6):917-923.

13. Bauer WR, Schulten $\mathrm{K}$ : Theory of contrast agents in magnetic resonance imaging: coupling of spin relaxation and transport. Magn Reson Med 1992, 26(1):16-39.

14. Kober F, Itis I, Cozzone PJ, Bernard M: Myocardial blood flow mapping in mice using high-resolution spin labeling magnetic resonance imaging: influence of ketamine/xylazine and isoflurane anesthesia. Magn Reson Med 2005, 53(3):601-606.

15. Detre JA, Leigh JS, Williams DS, Koretsky AP: Perfusion imaging. Magn Reson Med 1992, 23(1):37-45.

16. Richer C, Domergue V, Gervais M, Bruneval P, Giudicelli JF: Fluospheres for cardiovascular phenotyping genetically modified mice. J Cardiovasc Pharmacol 2000, 36(3):396-404
17. Vandsburger $M H$, Janiczek $R L, X u$, French BA, Meyer $C H$, Kramer $C M$, Epstein $\mathrm{FH}$ : Improved arterial spin labeling after myocardial infarction in mice using cardiac and respiratory gated look-locker imaging with fuzzy C-means clustering. Magn Reson Med 2010, 63(3):648-657

18. Makowski M, Jansen C, Webb I, Chiribiri A, Nagel E, Botnar R, Kozerke S, Plein S: First-pass contrast-enhanced myocardial perfusion MRI in mice on a 3-T clinical MR scanner. Magn Reson Med 2010, 64(6):1592-1598

19. Nahrendorf M, Streif JU, Hiller KH, Hu K, Nordbeck P, Ritter O, Sosnovik D, Bauer L, Neubauer S, Jakob PM, et al.: Multimodal functional cardiac MRI in creatine kinase-deficient mice reveals subtle abnormalities in myocardial perfusion and mechanics. Am J Physiol Heart Circ Physiol 2006, 290(6):H2516- $\mathrm{H} 2521$

20. Vandsburger MH, French BA, Helm PA, Roy RJ, Kramer CM, Young AA, Epstein FH: Multi-parameter in vivo cardiac magnetic resonance imaging demonstrates normal perfusion reserve despite severely attenuated beta-adrenergic functional response in neuronal nitric oxide synthase knockout mice. Eur Heart J 2007, 28(22):2792-2798.

\section{doi:10.1186/1532-429X-14-73}

Cite this article as: Abeykoon et al:: Quantitative myocardial perfusion in mice based on the signal intensity of flow sensitized CMR. Journal of Cardiovascular Magnetic Resonance 2012 14:73.

\section{Submit your next manuscript to BioMed Central and take full advantage of:}

- Convenient online submission

- Thorough peer review

- No space constraints or color figure charges

- Immediate publication on acceptance

- Inclusion in PubMed, CAS, Scopus and Google Scholar

- Research which is freely available for redistribution

Submit your manuscript at www.biomedcentral.com/submit
C Biomed Central 